\title{
Gauge Coupling Unification and Light Exotica in String Theory
}

\author{
Stuart Raby and Akın Wingerter \\ Department of Physics, The Ohio State University, 191 W. Woodruff Ave., Columbus, Ohio 43210, USA
}

(Received 2 May 2007; published 1 August 2007)

\begin{abstract}
In this Letter we consider the consequences for the CERN Large Hadron Collider of light vectorlike exotica with fractional electric charge. It is shown that such states are found in orbifold constructions of the heterotic string. Moreover, these exotica are consistent with gauge coupling unification at one loop, even though they do not come in complete multiplets of SU(5).
\end{abstract}

DOI: 10.1103/PhysRevLett.99.051802

The Large Hadron Collider (LHC) era is about to begin. The minimal supersymmetric standard model (MSSM) is the premier candidate for new physics beyond the standard model (SM), providing a plethora of new signatures for LHC physics. Many attempts have been made to derive the MSSM from string theory. Such attempts typically lead to the MSSM spectrum plus additional states, termed exotics.

D-brane constructions typically contain exotics which are chiral under the standard model gauge group [1]. As a result, in the best of circumstances these chiral exotics obtain mass of order of the weak scale, only after electroweak symmetry breaking. In the worst of circumstances, the chiral exotics remain massless. Hence these theories with chiral exotics are severely constrained by precision electroweak data [2].

Recently, heterotic string constructions using orbifold compactifications have provided models which have only the MSSM spectrum or the MSSM spectrum with the addition of vectorlike exotics [3-10]. Vectorlike exotics can, by definition, obtain mass at an arbitrary scale without breaking any standard model gauge symmetry. Simple examples of vectorlike exotics include additional pairs of Higgs doublets $\left(\mathbf{H}_{\mathbf{u}}+\mathbf{H}_{\mathbf{d}}\right)$, a pair of states with the quantum numbers of a down quark, $\mathbf{D}^{\mathbf{c}},(\overline{3}, 1)_{2 / 3}$ and its charge conjugate or complete multiplets under SU(6) such as $\overline{\mathbf{5}}+$ $\mathbf{5}$, transforming under the SM as $(\overline{\mathbf{3}}, \mathbf{1})_{\mathbf{2} / \mathbf{3}}+(\mathbf{1}, \mathbf{2})_{-1}\left[\mathbf{D}^{\mathbf{c}}+\right.$ L] + c.c. In the first example, these states contribute to the renormalization group running of the SM gauge coupling in such a way as to change the grand unified theory (GUT) scale. If these states are light, with mass of order the weak scale, then they will destroy the nice agreement of the LEP data and gauge coupling unification in supersymmetric (SUSY) GUTs. Hence, in order to preserve the nice features of SUSY GUTs, one typically argues that these states have mass of order the GUT scale. On the other hand, if the vectorlike exotics come in complete $\mathrm{SU}(5)$ multiplets, then at least at one loop they do not affect gauge coupling unification, even if they are light. In fact, light vectorlike exotics in complete SU(5) multiplets have been used as messengers for low energy gauge-mediated SUSY breaking [11-14].

PACS numbers: 12.10.Dm, 11.25.Wx, 12.60.Jv, 14.80.-j

In this Letter we point out that it is also possible to have light vectorlike exotics, which are not in complete SU(5) multiplets, however, still preserving the nice features of SUSY GUTs $[15,16]$. Moreover, in the simple example, these states have fractional electric charge and thus it would be very interesting to search for such states at the LHC. Such states are present in many heterotic orbifold constructions.

Stringy exotica. - In Refs. [3,5] the $E_{8} \times E_{8}$ heterotic string was compactified on a particular six torus modded out by the discrete symmetry $\mathbb{Z}_{6}-\mathrm{II}=\mathbb{Z}_{3} \times \mathbb{Z}_{2}$. By taking one compactified dimension to be much larger than the other 5 , it was shown in one of the examples that the $\mathbb{Z}_{3}$ orbifold by itself produced a low energy effective field theory (below the string scale) equivalent to an $E_{6}$ orbifold GUT in 5 space-time dimensions. The final $\mathbb{Z}_{2}$ orbifolding of the one extra dimension, along with one Wilson line, reduced the $E_{6}$ gauge symmetry to Pati-Salam (PS) $\mathrm{SU}(4) \times \mathrm{SU}(2)_{L} \times \mathrm{SU}(2)_{R}$. The resulting orbifold described a line segment with two end-of-the-world branes. At one end the gauge symmetry was $\mathrm{SO}(10)$, while at the other end it was $\mathrm{SU}(6) \times \mathrm{SU}(2)_{R}$. Whereas standard model families resided on the $\mathrm{SO}(10)$ brane, it was found that vectorlike exotics in the representations, $[(\mathbf{6}, \mathbf{1})+(\mathbf{1}, \mathbf{2})]+$ c.c., were localized on the $\mathrm{SU}(6) \times \mathrm{SU}(2)_{R}$ brane. Under PS, i.e.,

$$
\mathrm{SU}(6) \times \mathrm{SU}(2)_{R} \rightarrow \mathrm{SU}(4) \times \mathrm{SU}(2)_{L} \times \mathrm{SU}(2)_{R},
$$

these states transform as

$$
\begin{aligned}
{[(6,1)+(1,2)]+\text { c.c. } \rightarrow } & {[(4, \mathbf{1}, \mathbf{1})+(\mathbf{1}, \mathbf{2}, \mathbf{1})+(\mathbf{1}, \mathbf{1}, \mathbf{2})] } \\
& + \text { c.c. }
\end{aligned}
$$

Assuming that PS spontaneously breaks to the standard model, i.e.,

$\mathrm{SU}(4) \times \mathrm{SU}(2)_{L} \times \mathrm{SU}(2)_{R} \rightarrow \mathrm{SU}(3)_{c} \times \mathrm{SU}(2)_{L} \times \mathrm{U}(1)_{Y}$,

we finally obtain 


$$
\begin{aligned}
& {[(\mathbf{4}, \mathbf{1}, \mathbf{1})+(\mathbf{1}, \mathbf{2}, \mathbf{1})+(\mathbf{1}, \mathbf{1}, \mathbf{2})]+\text { c.c. }} \\
& \quad \rightarrow\left[(\mathbf{3}, \mathbf{1})_{1 / 3}+(\mathbf{1}, \mathbf{1})_{-1}+(\mathbf{1}, \mathbf{2})_{0}+(\mathbf{1}, \mathbf{1})_{ \pm 1}\right]+\text { c.c. } \\
& \quad=\left[\mathbf{Q}+\mathbf{E}_{-}+\mathbf{L}+\mathbf{E}_{ \pm}\right]+\text {c.c. }
\end{aligned}
$$

with $Y=(B-L)+2 T_{3 R}$ and electric charge $Q=T_{3 L}+$ $Y / 2$. Note, these states are explicitly vectorlike. They are all located in the same twisted sector of the string. Thus it is not unreasonable to expect that they all obtain mass (when SM singlet fields get supersymmetric vacuum expectation values) of the same order. We shall therefore explore the possibility that they have a gauge invariant, supersymmetric mass $\mathbf{M}$ of order of the electroweak scale (we assume here that all these exotics obtain the same mass $\mathbf{M}$; however, this is not a priori necessary). In addition, they are very exotic; henceforth we refer to them as exotica. They have fractional electric charge given by

$$
\left[\mathbf{Q}_{1 / 6}+\mathbf{E}_{-1 / 2}+\mathbf{L}_{ \pm 1 / 2}+\mathbf{E}_{ \pm 1 / 2}\right]+\text { c.c. }
$$

The exotic leptons have charges $\pm 1 / 2$, while the bound states of exotic quarks with SM quarks form an isovector and isoscalar multiplet of baryons

$$
\Sigma_{\mathrm{Q}}^{+3 / 2}, \Sigma_{\mathrm{Q}}^{+1 / 2}, \Sigma_{\mathrm{Q}}^{-1 / 2}, \Lambda_{\mathrm{Q}}^{+1 / 2},
$$

defined by

$$
[\mathbf{Q u u}]_{\mathbf{3} / \mathbf{2}},\left[\mathbf{Q}(\mathbf{u d})_{\mathbf{s}}\right]_{\mathbf{1} / \mathbf{2}},[\mathbf{Q d d}]_{-\mathbf{1} / \mathbf{2}},\left[\mathbf{Q}(\mathbf{u d})_{\mathbf{a}}\right]_{\mathbf{1} / \mathbf{2}},
$$

and an isodoublet of mesons

$$
\mathbf{Q}_{\mathbf{u}}^{+1 / 2}=[\overline{\mathbf{Q}} \mathbf{u}]_{+1 / 2}, \mathbf{Q}_{\mathbf{d}}^{-1 / 2}=[\overline{\mathbf{Q}} d]_{-1 / 2}
$$

Searches for fractionally charged heavy particles exclude them with mass less than $200 \mathrm{GeV}$ [17-19]. Nevertheless they can be produced at the Tevatron or the LHC via the Drell-Yan processes. The fermionic exotica are expected to be lighter than their scalar partners, due to soft SUSY breaking contributions to the scalar masses. Therefore the scalar exotica will decay to their fermionic partners and a gaugino [either a gluino, chargino, or neutralino depending on the quantum numbers of the (s)exotica]. Moreover, unless the flavor symmetries of the leptonic exotica are broken via Yukawa couplings to the MSSM Higgs bosons, they will all be stable. [Note, bounds on stable heavy hydrogen are very severe. For example, Ref. [20] (see also Ref. [21]) finds the relative abundance to baryons less than of order $10^{-28}$. Although these fractionally charged exotics are stable, they can annihilate. We do not consider the cosmological evolution of these states in this Letter.)

The lightest fractionally charged color singlet state will also be stable. However, due to exothermic processes (similar exothermic processes for $R$ hadrons was considered in Refs. [22-24]) such as

$$
\overline{\mathbf{Q}}_{\mathbf{d}}^{+1 / 2}+\mathbf{p} \rightarrow \Sigma_{\mathbf{Q}}^{+3 / 2}+\pi^{0}
$$

we would expect the baryonic exotica to be more abundant than the mesons. In addition, we expect the beta decay processes

$$
\Sigma_{\mathrm{Q}}^{-1 / 2} \rightarrow \Lambda_{\mathrm{Q}}^{+1 / 2}+\mathrm{e}^{-}+\bar{\nu}_{\mathrm{e}}
$$

to occur with the lifetime $\tau_{\mathbf{\Sigma}_{\mathbf{Q}}^{-1 / 2}} \sim 10^{-5} \mathrm{sec}$, of order $\tau_{\mathbf{\Sigma}^{+}} / \mathbf{B r}\left(\boldsymbol{\Sigma}^{+} \rightarrow \boldsymbol{\Lambda}^{\mathbf{0}}+\mathbf{e}^{+}+\nu_{\mathbf{e}}\right)$. Thus the $\mathbf{Q}$ s will be produced at the Tevatron or the LHC predominantly via gluons; they will hadronize and the color singlet bound state exotica will likely be stopped in the hadronic calorimeter. Note, the electric charge on one fractionally charged state cannot be screened. However two fractionally charged states can have their charges screened by the surrounding normal matter.

The novel feature of these exotica is that even though they do not come in complete representations of SU(5), they nevertheless preserve gauge coupling unification at one loop.

Gauge coupling unification. - Consider first the evaluation of $\sin ^{2}\left(\theta_{W}\right)$ at $M_{\mathrm{GUT}}$. In general, we have

$$
\left.\sin ^{2}\left(\theta_{W}\right)\right|_{M_{G}}=\frac{1}{1+C^{2}} \quad \text { with } \quad C^{2}=\frac{\operatorname{Tr}\left(Y^{2} / 4\right)}{\operatorname{Tr}\left(T_{3 L}^{2}\right)} .
$$

For example, if we take the trace over the 5-plet of $\mathrm{SU}(5)$ we find $C^{2}=5 / 3$ and $\left.\sin ^{2}\left(\theta_{W}\right)\right|_{M_{G}}=3 / 8$. However, we find the same value of $C^{2}=5 / 3$ for the exotica in Eq. (2). Hence the GUT boundary conditions are unchanged with the addition of these states.

Now consider RG running below the GUT scale. We show that the prediction of gauge coupling unification is unchanged. At one loop we have

$$
\frac{d \alpha_{i}}{d t}=-\frac{b_{i}}{2 \pi} \alpha_{i}^{2}, \quad t=\ln \left(\mu / \mu_{0}\right)
$$

with

$$
b_{i}=3 C_{2}\left(G_{i}\right)-n T(r) .
$$

In this equation, $n$ is the number of chiral multiplets in the representation $r$ of the gauge group $G_{i} . \operatorname{Tr}\left(T_{A} T_{B}\right)=$ $T(r) \delta_{A B}$, where $T_{A}$ is the gauge generator for the chiral multiplet. $T(r)=1 / 2$ for the $N$ dimensional representation of $\mathrm{SU}(N)$ and $T(r)=(3 / 5) Y^{2} / 4$ for $\mathrm{U}(1)_{Y}$. Finally, for $\mathrm{SU}(N)$, the quadratic Casimir operator for the adjoint representation, $C_{2}[\mathrm{SU}(N)]=N$.

Let $\Delta b_{i}$ be the contribution of the exotica to the RG running parameters, Eq. (5). We find $\Delta b_{i}=-1$ for all $i$. Hence, gauge coupling unification is unaffected by these states at one loop, even if they have weak scale masses; i.e., the value of the GUT scale and the prediction of $\alpha_{s}\left(M_{Z}\right)$ in terms of $\alpha\left(M_{Z}\right)$ and $\sin ^{2} \theta\left(M_{Z}\right)$ are unchanged. In fact, the only effect of these states is to increase the value of the grand unified gauge coupling, $\alpha_{G}$.

One may inquire whether these novel exotica are generic in string model constructions. We have looked at the 
exotica found in the "mini-landscape" search of Ref. [10]. In many cases (of order 5\%) we find exotica with properties similar to those in Eq. (2). However, unlike the previous exotica, these states transform nontrivially under a hidden sector gauge group. Consider the following two examples:

$$
\begin{aligned}
& 2\left[(\overline{\mathbf{3}}, \mathbf{1}, \mathbf{1})_{-1 / 3}+(\mathbf{1}, \mathbf{1}, \mathbf{1})_{1}+(\mathbf{1}, \mathbf{2}, \mathbf{1})_{0}+(\mathbf{1}, \mathbf{1}, \mathbf{1})_{ \pm 1}\right] \\
& \quad+2\left[(\mathbf{3}, \mathbf{1}, \mathbf{1})_{1 / 3}+(\mathbf{1}, \mathbf{1}, \mathbf{1})_{-1}+(\mathbf{1}, \mathbf{2}, \mathbf{1})_{0}\right]+(\mathbf{1}, \mathbf{1}, \mathbf{2})_{ \pm 1}
\end{aligned}
$$

transforming under

$$
\mathrm{SU}(3)_{c} \times \mathrm{SU}(2)_{L} \times \mathrm{SU}(2)_{A} \times \mathrm{U}(1)_{Y},
$$

and

$$
\begin{aligned}
& {\left[(\mathbf{3}, \mathbf{2}, \mathbf{1}, \mathbf{1})_{1 / 3}+5(\overline{\mathbf{3}}, \mathbf{1}, \mathbf{1}, \mathbf{1})_{2 / 3}+2(\mathbf{1}, \mathbf{2}, \mathbf{2}, \mathbf{1})_{0}\right.} \\
& \left.\quad+4(\mathbf{1}, \mathbf{1}, \mathbf{2}, \mathbf{1})_{ \pm 1}\right]+\left[(\overline{\mathbf{3}}, \mathbf{2}, \mathbf{1}, \mathbf{1})_{-1 / 3}+5(\mathbf{3}, \mathbf{1}, \mathbf{1}, \mathbf{1})_{-2 / 3}\right. \\
& \left.\quad+2(\mathbf{1}, \mathbf{2}, \mathbf{1}, \mathbf{2})_{0}+4(\mathbf{1}, \mathbf{1}, \mathbf{1}, \mathbf{2})_{ \pm 1}\right]
\end{aligned}
$$

transforming under

$$
\mathrm{SU}(3)_{c} \times \mathrm{SU}(2)_{L} \times \mathrm{SU}(2)_{A} \times \mathrm{SU}(2)_{B} \times \mathrm{U}(1)_{Y} .
$$

These states can all be given a supersymmetric mass $\mathbf{M}$ without breaking any gauge symmetries. Moreover, the low energy theory is anomaly free. Although the exotica do not affect the value of the GUT scale or the low energy prediction from gauge coupling unification at one loop, they do increase the value of $\alpha_{\mathrm{GUT}}$. In the first example [Eq. (2)] we find $\alpha_{\mathrm{GUT}}^{-1}=19$. In the second example [Eq. (6)] we find $\alpha_{\text {GUT }}^{-1}=14$. These two cases are consistent with perturbative unification. However, in the third example [Eq. (7)], there is a problem since we reach the Landau pole before the GUT scale. Hence, if we demand perturbative unification, this last case is excluded.

In these examples, the hidden SU(2) gauge symmetry can become strong forming $\mathrm{SU}(2)$ singlet bound states. We consider a more general example below.

Exotica with hidden sector charge.-As discussed in the above examples it is possible in string theory for the exotica to transform nontrivially under a hidden sector gauge group. Here we consider a generalized example, not obtained from a particular string construction, which has interesting phenomenology. Consider a hidden sector gauge group $\mathrm{SU}(N)$ with the exotica transforming as

$$
[(6,1, N)+(1,2, N)]+\text { c.c. }
$$

under $\mathrm{SU}(6) \times \mathrm{SU}(2)_{R} \times \mathrm{SU}(N)$, or as

$$
[(4,1,1, N)+(1,2,1, N)+(1,1,2, N)]+\text { c.c. }
$$

under $\mathrm{SU}(4) \times \mathrm{SU}(2)_{L} \times \mathrm{SU}(2)_{R} \times \mathrm{SU}(N)$. Note, pursuant to the previous section, values of $N>3$ are excluded by demanding perturbative unification.

Assuming the hidden sector gauge coupling gets strong at a scale $\Lambda_{N} \gg M_{Z}$, the exotica will form $\mathrm{SU}(N)$ singlet bound states with mass of order $\Lambda_{N}$. Once again these states will not affect gauge coupling unification at one loop. The phenomenology of such $\mathrm{SU}(N)$ singlet "baryons" and "mesons" will depend on the values of $N$ and $\Lambda_{N}$.

At the scale $\Lambda_{N}$ we can expect the $N$ exotica [Eq. (8)] and their charge conjugates to form chiral condensates (unlike technicolor theories, these chiral condensates do not break the standard model gauge symmetries). Neglecting the standard model gauge interactions, this theory has a $\mathrm{U}(8)_{L} \times \mathrm{U}(8)_{R}$ chiral symmetry which is broken to $\mathrm{U}(8)_{\text {vector }}$ via the condensates. Hence we obtain 64 pseudo Nambu-Goldstone (PNG) bosons, expected to be the lightest particles of the strong $\mathrm{SU}(N)$ gauge interactions. For an analysis of the PNG spectrum, see [25-27]. Most of these states transform nontrivially under QCD and are expected to have mass $m \approx\left(\frac{C_{2}(r) \alpha_{s}\left(\Lambda_{N}\right)}{\alpha_{E M}}\right) \times$ $\frac{\left(m_{\pi^{+}}^{2}-m_{\pi^{0}}^{2}\right)^{1 / 2}}{\Lambda_{\text {QCD }}} \Lambda_{N} \sim \Lambda_{N}$, where $C_{2}(r)$ is the quadratic Casimir for the pseudo-NG boson in representation $r$ of QCD. On the other hand, some states with only electroweak quantum numbers will be much lighter. These states typically have mass squared of order

$$
m^{2} \approx \alpha_{2}\left(\Lambda_{N}\right) \Lambda_{N}^{2} .
$$

However, a few of them [i.e., bound states $[(\mathbf{1}, \mathbf{2}, \mathbf{1}, \overline{\mathbf{N}}) \otimes$ $(\mathbf{1}, \mathbf{1}, \mathbf{2}, \mathbf{N})]$ under Pati-Salam $\times \mathrm{SU}(N)]$ have mass squared

$$
m^{2} \approx \frac{3 \alpha}{4 \pi} M_{Z}^{2} \ln \left(\Lambda_{N}^{2} / M_{Z}^{2}\right) .
$$

These states have identical electroweak quantum numbers to the pair of Higgs doublets in the MSSM, i.e., $\check{\boldsymbol{H}}_{u}, \check{\boldsymbol{H}}_{d}$ and, hopefully without confusion, we use this notation to apply to these exotica here. Finally the lightest states are axionlike with no standard model gauge quantum numbers.

These light states significantly constrain the lower bound on $\Lambda_{N}$. In fact, bounds on an invisible axion would apply here and we would therefore expect $\Lambda_{N}>10^{8} \mathrm{GeV}$, from astrophysical constraints on an invisible axion [28]. However, the charged states $\check{\boldsymbol{H}}_{u}^{+}$and $\check{\boldsymbol{H}}_{d}^{-}$would have mass less than $M_{Z}$ even for $\Lambda_{N} \sim M_{\text {GUT }}$ [see Eq. (11)]. They would most likely have been observed at LEP or the Tevatron via the Drell-Yan production of the exotica through an off-shell photon or $Z$. The only way out is to also give these exotica [Eq. (8)] a gauge invariant mass $\mathbf{M} \geq 200 \mathrm{GeV}$. Then all the $\mathrm{SU}(N)$ gauge singlets get mass of order $\mathbf{M}$.

We then can consider two possibilities, either $\Lambda_{N} \geq \mathbf{M}$ or $\Lambda_{N} \ll \mathbf{M}$. The first case is comparable to QCD with all quark masses less than or equal to $\Lambda_{\mathrm{QCD}}$. The PNG bosons will then have two contributions to their mass squared, i.e., the radiative contributions considered previously and the explicit mass contribution given by $\delta m^{2}=\mathbf{M} \Lambda_{N}^{3} / F_{N}^{2}$, where $F_{N}$ is the axion decay constant. A more novel 
scenario occurs if we take $\Lambda_{N} \ll \mathbf{M}$. These exotica have properties similar to the "quirks" introduced in Ref. [29]. They can be produced at the LHC. When the exotics are produced they can separate by large distances in the detector before forming the bound state, since their effective string tension is so much smaller than their mass. In addition, we expect $\check{\boldsymbol{H}}_{u}^{+}$and $\check{\boldsymbol{H}}_{d}^{-}$to be heavier than their neutral weak doublet partners $\check{\boldsymbol{H}}_{u}^{0}$ and $\check{\boldsymbol{H}}_{d}^{0}$. Thus the charged states will decay via the beta process $\check{\boldsymbol{H}}_{d}^{-} \rightarrow \check{\boldsymbol{H}}_{d}^{0}+$ $e+\bar{\nu}_{e}$. The neutral ones are stable and may be dark matter candidates (these neutral states would be comparable to the $K^{0} \mathrm{~s}$ in the standard model, assuming strangeness was conserved). Finally, the lightest $\mathrm{SU}(N)$ singlet exotics can be defined as follows. Let $\Psi=(8, N)$ define a Dirac spinor for the exotica of Eq. (8) in an $\mathrm{SU}(8) \times \mathrm{SU}(N)$ notation. Then the axial currents $J_{\mu 5}^{A}=\bar{\Psi} \gamma_{\mu} \gamma_{5} T^{A} \Psi$, with $T^{A}$ given by the 4 generators in the Cartan subalgebra of $\mathrm{SU}(8)$ but not in $\mathrm{SU}(3)_{c} \times \mathrm{SU}(2)_{L}$, create these light PNG bosons from the vacuum. Two of these can either decay to two photons or two gluons through the triangle anomaly. Clearly, all these exotica would have very interesting signatures at the LHC.

Conclusion. - It is well known that the prediction of gauge coupling unification in traditional SUSY GUTs is unaffected at one loop by the presence of light vectorlike states in complete multiplets of SU(5). In this Letter we have identified a class of vectorlike exotica which (i) do not come in complete SU(5) multiplets, (ii) do not affect gauge coupling unification at one loop, (iii) can have fractional electric charge, and (iv) are found in orbifold constructions of the heterotic string. Such states provide an interesting challenge for the LHC.

We would like to acknowledge research supported in part by the Department of Energy under Grant No. DOE/ ER/01545-872.

[1] R. Blumenhagen, M. Cvetic, P. Langacker, and G. Shiu, Annu. Rev. Nucl. Part. Sci. 55, 71 (2005).

[2] W.-M. Yao et al., J. Phys. G 33, 1 (2006).

[3] T. Kobayashi, S. Raby, and R. J. Zhang, Phys. Lett. B 593, 262 (2004).

[4] S. Förste et al., Phys. Rev. D 70, 106008 (2004).

[5] T. Kobayashi, S. Raby, and R. J. Zhang, Nucl. Phys. B704, 3 (2005).

[6] W. Buchmüller et al., Phys. Rev. Lett. 96, 121602 (2006).

[7] W. Buchmüller et al., arXiv:hep-th/0606187.
[8] W. Buchmüller et al., Nucl. Phys. B712, 139 (2005).

[9] W. Buchmüller et al., arXiv:hep-ph/0512326.

[10] O. Lebedev, H. P. Nilles, S. Raby, S. Ramos-Sanchez, M. Ratz, P. K. S. Vaudrevange, and A. Wingerter, Phys. Lett. B 645, 88 (2007).

[11] M. Dine, W. Fischler, and M. Srednicki, Nucl. Phys. B189, 575 (1981); S. Dimopoulos and S. Raby, Nucl. Phys. B192, 353 (1981); M. Dine and W. Fischler, Phys. Lett. B 110, 227 (1982); M. Dine and M. Srednicki, Nucl. Phys. B202, 238 (1982); L. Alvarez-Gaume, M. Claudson, and M. Wise, Nucl. Phys. B207, 96 (1982); C. Nappi and B. Ovrut, Phys. Lett. B 113, 175 (1982).

[12] M. Dine and W. Fischler, Nucl. Phys. B204, 346 (1982); S. Dimopoulos and S. Raby, Nucl. Phys. B219, 479 (1983).

[13] M. Dine, A. E. Nelson, and Y. Shirman, Phys. Rev. D 51, 1362 (1995); M. Dine, A.E. Nelson, Y. Nir, and Y. Shirman, Phys. Rev. D 53, 2658 (1996).

[14] G. F. Giudice and R. Rattazzi, Phys. Rep. 322, 419 (1999).

[15] S. Dimopoulos, S. Raby, and F. Wilczek, Phys. Rev. D 24, 1681 (1981); S. Dimopoulos and H. Georgi, Nucl. Phys. B193, 150 (1981); L. Ibanez and G. G. Ross, Phys. Lett. B 105, 439 (1981); N. Sakai, Z. Phys. C 11, 153 (1981); M. B. Einhorn and D. R. T. Jones, Nucl. Phys. B196, 475 (1982); W. J. Marciano and G. Senjanovic, Phys. Rev. D 25, 3092 (1982).

[16] See review article, S. Raby et al. (Particle Data Group), J. Phys. G 33, 1 (2006).

[17] D. Acosta et al. (CDF Collaboration), Phys. Rev. Lett. 90, 131801 (2003).

[18] M. L. Perl, E. R. Lee, and D. Loomba, Mod. Phys. Lett. A 19, 2595 (2004).

[19] M. Fairbairn, A. C. Kraan, D. A. Milstead, T. Sjostrand, P. Skands, and T. Sloan, Phys. Rep. 438, 1 (2007).

[20] P. F. Smith et al., Nucl. Phys. B206, 333 (1982).

[21] M. L. Perl, P. C. Kim, V. Halyo, E. R. Lee, I. T. Lee, D. Loomba, and K. S. Lackner, Int. J. Mod. Phys. A 16, 2137 (2001).

[22] A. C. Kraan, Eur. Phys. J. C 37, 91 (2004).

[23] A. C. Kraan, arXiv:hep-ex/0506009.

[24] A. Arvanitaki, S. Dimopoulos, A. Pierce, S. Rajendran, and J. G. Wacker, arXiv:hep-ph/0506242 [Phys. Rev. D (to be published)].

[25] S. Dimopoulos, Nucl. Phys. B168, 69 (1980).

[26] S. Dimopoulos, L. Susskind, and S. Raby, AIP Conference Proceedings, Particles and Field, Montreal, 1979 (AIP, New York, 1980), Vol. 59, No. 1, p. 407.

[27] S. Dimopoulos, S. Raby, and G. L. Kane, Nucl. Phys. B182, 77 (1981).

[28] P. Sikivie, AIP Conf. Proc. 805, 23 (2005).

[29] J. Kang, M. A. Luty, and S. Nasri, arXiv:hep-ph/0611322. 